\title{
ON BALANCED SUBGROUPS OF THE MULTIPLICATIVE GROUP
}

\author{
CARL POMERANCE AND DOUGLAS ULMER \\ In memory of Alf van der Poorten
}

\begin{abstract}
A subgroup $H$ of $G=(\mathbb{Z} / d \mathbb{Z})^{\times}$is called balanced if every coset of $H$ is evenly distributed between the lower and upper halves of $G$, i.e., has equal numbers of elements with representatives in $(0, d / 2)$ and $(d / 2, d)$. This notion has applications to ranks of elliptic curves. We give a simple criterion in terms of characters for a subgroup $H$ to be balanced, and for a fixed integer $p$, we study the distribution of integers $d$ such that the cyclic subgroup of $(\mathbb{Z} / d \mathbb{Z})^{\times}$generated by $p$ is balanced.
\end{abstract}

\section{INTRODUCTION}

Let $d>2$ be an integer and consider $\mathbb{U}_{d}=(\mathbb{Z} / d \mathbb{Z})^{\times}$, the group of units modulo $d$. Let $A_{d}$ be the first half of $\mathbb{U}_{d}$; that is, $A_{d}$ consists of residues with a representative in $(0, d / 2)$. Let $B_{d}=\mathbb{U}_{d} \backslash A_{d}$ be the second half of $\mathbb{U}_{d}$. We say a subgroup $H$ of $\mathbb{U}_{d}$ is balanced if for each $u \in \mathbb{U}_{d}$ we have $\left|u H \cap A_{d}\right|=\left|u H \cap B_{d}\right|$; that is, each coset of $H$ has equally many members in the first half of $\mathbb{U}_{d}$ as in the second half.

Our interest in this notion stems from the following result.

Theorem 1.1 ([2]). Let $p$ be an odd prime number, let $\mathbb{F}_{q}$ be the finite field of cardinality $q=p^{f}$, and let $\mathbb{F}_{q}(u)$ be the rational function field over $\mathbb{F}_{q}$. Let $d$ be a positive integer not divisible by $p$, and for e a divisor of $d$ write $\langle p\rangle_{e}$ for the cyclic subgroup of $(\mathbb{Z} / e \mathbb{Z})^{\times}$generated by $p$. Let $E_{d}$ be the elliptic curve over $\mathbb{F}_{q}(u)$ defined by

$$
y^{2}=x(x+1)\left(x+u^{d}\right) .
$$

Then we have

$$
\operatorname{Rank} E_{d}\left(\mathbb{F}_{q}(u)\right)=\sum_{\substack{e \mid d \\ e>2}} \begin{cases}\frac{\varphi(e)}{l_{q}(e)} & \text { if }\langle p\rangle_{e} \text { is balanced, } \\ 0 & \text { if not. }\end{cases}
$$

Here $\varphi$ is Euler's function and $l_{q}(e)$ is the order of $q$ in $(\mathbb{Z} / e \mathbb{Z})^{\times}$.

A few simple observations are in order. It is easy to see that $\langle-1\rangle$ is a balanced subgroup of $\mathbb{U}_{d}$. It is also easy to see that if $4 \mid d$, then $\left\langle\frac{1}{2} d+1\right\rangle$ is a balanced subgroup of $\mathbb{U}_{d}$. In addition, if $H$ is a balanced subgroup of $\mathbb{U}_{d}$ and $K$ is a subgroup of $\mathbb{U}_{d}$ containing $H$, then $K$ is balanced as well. Indeed, $K$ is a union of $[K: H]$ cosets of $H$, so for each $u \in \mathbb{U}_{d}, u K$ is a union of $[K: H]$ cosets of $H$, each equally distributed between the first half of $\mathbb{U}_{d}$ and the second half. Thus, $u K$ is also equally distributed between the first half and the second half.

2010 Mathematics Subject Classification. Primary 11N37; Secondary 11G05.

The first author was partially supported by NSF grant DMS-1001180. 
It follows that if some power of $p$ is congruent to -1 modulo $d$ and if $q \equiv 1(\bmod d)$, then the theorem implies that $\operatorname{Rank} E\left(\mathbb{F}_{q}(u)\right)=d-2$ if $d$ is even and $d-1$ if $d$ is odd. The rank of $E$ when some power of $p$ is -1 modulo $d$ was first discussed in [12], and with hindsight it could have been expected to be large from considerations of "supersingularity." The results of [2] show, perhaps surprisingly, that there are many other classes of $d$ for which high ranks occur. Our aim here is to make this observation more quantitative.

More precisely, the aim of this paper is to investigate various questions about balanced pairs $(p, d)$, i.e., pairs such that $\langle p\rangle$ is a balanced subgroup of $(\mathbb{Z} / d \mathbb{Z})^{\times}$. In particular, we give a simple criterion in terms of characters for $(p, d)$ to be balanced (Theorem 2.1), and we use it to determine all balanced subgroups of order 2 (Theorem 3.2). We also investigate the distribution for a fixed $p$ of the set of $d$ 's such that $(p, d)$ is balanced (Theorems 4.1, 4.2). We find that when $p$ is odd, the divisors of $p^{n}+1$ are not the largest contributor to this set. Finally, we investigate the average rank and typical rank given by Theorem 1.1 for fixed $q$ and varying $d$.

\section{BALANCED SUBGROUPS AND CHARACTERS}

In this section we write $G$ (rather than $\left.\mathbb{U}_{d}\right)$ for $(\mathbb{Z} / d \mathbb{Z})^{\times}$. We also write $A$ for $A_{d}$ as above and similarly for $B$, so that $G$ is the disjoint union $A \cup B$.

We write $\mathbf{1}_{A}$ for the characteristic function of $A \subset G$ and similarly for $\mathbf{1}_{B}$. Let $f: G \rightarrow \mathbb{C}$ be the sum over $H$ of translates of $\mathbf{1}_{A}-\mathbf{1}_{B}$ :

$$
\begin{aligned}
f(g) & =\sum_{h \in H}\left(\mathbf{1}_{A}(g h)-\mathbf{1}_{B}(g h)\right) \\
& =\#(g H \cap A)-\#(g H \cap B) .
\end{aligned}
$$

By definition, $H$ is balanced if and only if $f$ is identically zero.

We write $\hat{G}$ for the set of complex characters of $G$, and we expand $f$ in terms of these characters:

$$
f=\sum_{\chi \in \hat{G}} \hat{f}(\chi) \chi
$$

where

$$
\hat{f}(\chi)=\frac{1}{\varphi(d)} \sum_{g \in G} f(g) \chi^{-1}(g) .
$$

It is easy to see that $\hat{f}\left(\chi_{\text {triv }}\right)=0$. Noting that $\mathbf{1}_{A}-\mathbf{1}_{B}=2 \mathbf{1}_{A}-\mathbf{1}_{G}$, for $\chi$ non-trivial we find that

$$
\hat{f}\left(\chi^{-1}\right)=\frac{2}{\varphi(d)}\left(\sum_{h \in H} \chi(h)\right)\left(\sum_{a \in A} \chi(a)\right) .
$$

Note that $\sum_{h \in H} \chi(h)$ is zero if and only if the restriction of $\chi$ to $H$ is non-trivial. We introduce the notation

$$
c_{\chi}=\sum_{a \in A} \chi(a)
$$

We view $\chi$ as a Dirichlet character, so that we also have

$$
c_{\chi}=\sum_{0<a<d / 2} \chi(a) \text {. }
$$


As usual, we say $\chi$ is even if $\chi(-1)=1$ and $\chi$ is odd if $\chi(-1)=-1$. Note that if $\chi$ is even and non-trivial, then

$$
c_{\chi}=\frac{1}{2} \sum_{g \in G} \chi(g)=0 .
$$

This discussion yields the following characterization of balanced subgroups:

Theorem 2.1. With notation as above, we have that $H$ is balanced if and only if $c_{\chi}=0$ for every odd character $\chi$ of $G$ whose restriction to $H$ is trivial.

As an example, note that if $H=\langle-1\rangle$, then there are no odd characters trivial on $H$ and so the theorem implies that $H$ is balanced.

We now give a non-vanishing criterion for $c_{\chi}$.

Lemma 2.2. If $\chi$ is a primitive, odd character of $G$, then $c_{\chi} \neq 0$.

Proof. Under the hypotheses on $\chi$, the classical evaluation of $L(1, \chi)$ leads to the formula

$$
L\left(1, \chi^{-1}\right)=\frac{\pi i \tau\left(\chi^{-1}\right)}{d\left(\chi^{-1}(2)-2\right)} c_{\chi}
$$

where $\tau\left(\chi^{-1}\right)$ is a Gauss sum. (See, e.g., [7, pp. 200-201] or [8, Theorem 9.21], though there is a small typo in the second reference.) By the theorem of Dirichlet, $L\left(1, \chi^{-1}\right) \neq 0$ and so $c_{\chi} \neq 0$.

In light of the lemma, we should consider imprimitive characters.

Lemma 2.3. Suppose that $\ell$ is a prime number dividing $d$ and set $d^{\prime}=d / \ell$. Suppose also that $\chi$ is a non-trivial character modulo d induced by a character $\chi^{\prime}$ modulo $d^{\prime}$. If $\ell=2$, then $c_{\chi}=-\chi^{\prime}(2) c_{\chi^{\prime}}$. If $\ell$ is odd, then $c_{\chi}=\left(1-\chi^{\prime}(\ell)\right) c_{\chi^{\prime}}$. Here we employ the usual convention that $\chi^{\prime}(\ell)=0$ if $\ell \mid d^{\prime}$.

Proof. First suppose $\ell=2$. We have

$$
c_{\chi}=\sum_{\substack{a<d / 2 \\ \operatorname{gcd}(a, d)=1}} \chi(a)=\sum_{\substack{a<d^{\prime} \\ \operatorname{gcd}\left(a, 2 d^{\prime}\right)=1}} \chi^{\prime}(a) .
$$

If $2 \mid d^{\prime}$, this is a complete character sum and so vanishes. If $2 \nmid d^{\prime}$, then

$$
\begin{aligned}
\sum_{\substack{a<d^{\prime} \\
\operatorname{gcd}\left(a, 2 d^{\prime}\right)=1}} \chi^{\prime}(a) & =\sum_{\substack{a<d^{\prime} \\
\operatorname{gcd}\left(a, d^{\prime}\right)=1}} \chi^{\prime}(a)-\sum_{\substack{a<d^{\prime} / 2 \\
\operatorname{gcd}\left(a, d^{\prime}\right)=1}} \chi^{\prime}(2 a) \\
& =-\sum_{\substack{a<d^{\prime} / 2 \\
\operatorname{gcd}\left(a, d^{\prime}\right)=1}} \chi^{\prime}(2 a) \\
& =-\chi^{\prime}(2) c_{\chi^{\prime}}
\end{aligned}
$$

as desired.

Now assume that $\ell$ is odd. We have

$$
c_{\chi}=\sum_{\substack{a<d / 2 \\ \operatorname{gcd}(a, d)=1}} \chi(a)=\sum_{\substack{a<\ell d^{\prime} / 2 \\ \operatorname{gcd}\left(a, \ell d^{\prime}\right)=1}} \chi^{\prime}(a) .
$$


If $\ell \mid d^{\prime}$, then

$$
\sum_{\substack{a<\ell d^{\prime} / 2 \\ \operatorname{gcd}\left(a, \ell d^{\prime}\right)=1}} \chi^{\prime}(a)=\sum_{\substack{a<d^{\prime} / 2 \\ \operatorname{gcd}\left(a, d^{\prime}\right)=1}} \chi^{\prime}(a)=c_{\chi^{\prime}} .
$$

If $\ell \backslash d^{\prime}$, then

$$
\begin{aligned}
\sum_{\substack{a<\ell d^{\prime} / 2 \\
\operatorname{gcd}\left(a, \ell d^{\prime}\right)=1}} \chi^{\prime}(a) & =\sum_{\substack{a<\ell d^{\prime} / 2 \\
\operatorname{gcd}\left(a, d^{\prime}\right)=1}} \chi^{\prime}(a)-\sum_{\substack{a<d^{\prime} / 2 \\
\operatorname{gcd}\left(a, d^{\prime}\right)=1}} \chi^{\prime}(\ell a) \\
& =\sum_{\substack{a<d^{\prime} / 2 \\
\operatorname{gcd}\left(a, d^{\prime}\right)=1}} \chi^{\prime}(a)-\chi^{\prime}(\ell) \sum_{\substack{a<d^{\prime} / 2 \\
\operatorname{gcd}\left(a, d^{\prime}\right)=1}} \chi^{\prime}(a) \\
& =\left(1-\chi^{\prime}(\ell)\right) c_{\chi^{\prime}}
\end{aligned}
$$

as desired.

Applying the lemma repeatedly, we arrive at the following non-vanishing criterion.

Proposition 2.4. Suppose that $\chi$ is an odd character modulo $d$ induced by a primitive character $\chi^{\prime}$ modulo $d^{\prime}$. Then $c_{\chi} \neq 0$ if and only if the following two conditions both hold: (i) $4 \chi \chi d$ or $d / d^{\prime}$ is odd; and (ii) for every odd prime $\ell$ which divides $d$ and does not divide $d^{\prime}$, we have $\chi^{\prime}(\ell) \neq 1$.

As an example, suppose that $4 \mid d$ and $H=\left\langle\frac{1}{2} d+1\right\rangle$. Note that

$$
(\mathbb{Z} / d \mathbb{Z})^{\times} /\left\langle\frac{1}{2} d+1\right\rangle \cong\left(\mathbb{Z} / \frac{1}{2} d \mathbb{Z}\right)^{\times}
$$

Thus, if $\chi$ is an odd character modulo $d$ and $\chi\left(\frac{1}{2} d+1\right)=1$, then the conductor $d^{\prime}$ of $\chi$ divides $d / 2$. This shows that $d / d^{\prime}$ is even and so condition (i) of the proposition fails and $c_{\chi}=0$. Therefore $H$ is balanced.

\section{BALANCED SUBGROUPS OF SMALL ORDER}

In this section, we discuss balanced subgroups of small order. We have already seen that a subgroup of $G$ which contains -1 or $\frac{1}{2} d+1$ is balanced. We will show that in a certain sense small balanced subgroups are mainly controlled by these balanced subgroups of order 2 .

Theorem 3.1. For every positive integer $n$ there is an integer $d(n)$ such that if $d>d(n)$ and $H$ is a balanced subgroup of $G=(\mathbb{Z} / d \mathbb{Z})^{\times}$of order $n$, then either $-1 \in H$ or $4 \mid d$ and $\frac{1}{2} d+1 \in H$.

We can make this much more explicit for subgroups of order 2:

Theorem 3.2. A subgroup $H=\langle h\rangle$ of order 2 is balanced if and only if $d$ and $h$ satisfy one of the following conditions:

(1) $h \equiv-1(\bmod d)$

(2) $d \equiv 0(\bmod 4)$ and $h \equiv \frac{1}{2} d+1(\bmod d)$

(3) $d=24$ and $h \equiv 17(\bmod d)$ or $h \equiv 19(\bmod d)$.

(4) $d=60$ and $h \equiv 41(\bmod d)$ or $h \equiv 49(\bmod d)$. 
Proof of Theorem 3.1. Using Proposition 2.4, we will show that if $d$ is sufficiently large with respect to $n$, then for any subgroup $H \subset(\mathbb{Z} / d \mathbb{Z})^{\times}$of order $n$ which does not contain -1 or $\frac{1}{2} d+1$, there is a character $\chi$ which is odd, trivial on $H$, and with $c_{\chi} \neq 0$. By Theorem 2.1, this implies that $H$ is not balanced.

Note that a balanced subgroup obviously has even order, so there is no loss in assuming that $n$ is even. We make this assumption for the rest of the proof.

Let $H^{+}$be the subgroup of $G$ generated by $H,-1$ and, if $4 \mid d$, by $\frac{1}{2} d+1$. Fix a character $\chi_{0}$ of $G$ which is trivial on $H$, odd, and -1 on $\frac{1}{2} d+1$ if $4 \mid d$. The set of all characters satisfying these restrictions is a homogeneous space for $\widehat{G / H^{+}} \subset \widehat{G}$. We will argue that multiplying $\chi_{0}$ by a suitable $\psi \in \widehat{G / H^{+}}$yields a $\chi=\chi_{0} \psi$ for which Proposition 2.4 implies that $c_{\chi} \neq 0$.

Note first that any character $\chi$ which is odd and, if $4 \mid d$, has $\chi\left(\frac{1}{2} d+1\right)=-1$ automatically satisfies condition (i) in Proposition 2.4. Indeed, if $4 \mid d$, then the condition $\chi\left(\frac{1}{2} d+1\right)=-1$ implies that $\chi$ is 2-primitive, i.e., the conductor $d^{\prime}$ of $\chi$ has $d / d^{\prime}$ odd. The rest of the argument relates to condition (ii) in Proposition 2.4.

Write $d=\prod_{\ell} \ell^{\ell_{\ell}}$ and write $G_{\ell}$ for $\left(\mathbb{Z} / \ell^{e_{\ell}} \mathbb{Z}\right)^{\times}$so that $G \cong \prod_{\ell} G_{\ell}$. Let $\chi=\prod_{\ell} \chi_{\ell}$. Note that $\ell$ divides the conductor of $\chi$ if and only if $\chi_{\ell}$ is non-trivial.

We will sloppily write $G_{\ell} / H^{+}$for $G_{\ell}$ modulo the image of $H^{+}$in $G_{\ell}$. For odd $\ell, G_{\ell}$ is cyclic and therefore so is $G_{\ell} / H^{+}$; for $\ell=2$, since $-1 \in H^{+}, G_{2} / H^{+}$is also cyclic.

Note also that $H^{+}$is the product of $H$ and a group of exponent 2, namely the subgroup of $G$ generated by -1 or by -1 and $\frac{1}{2} d+1$. Also, we have assumed that $n=|H|$ is even. If $\ell$ is odd, then $G_{\ell}$ is cyclic of even order, so has a unique element of order 2 . It follows that the order of the image of $H^{+}$in $G_{\ell}$ divides $n$.

We define three sets of odd primes:

$$
\begin{gathered}
S_{1}=\left\{\operatorname{odd} \ell: \ell \mid d, G_{\ell} / H^{+}=\{1\}\right\}, \\
S_{2}=\left\{\operatorname{odd} \ell: \ell\left|d, \varphi\left(\ell^{e_{\ell}}\right)\right| n\right\},
\end{gathered}
$$

and

$$
S_{3}=\{\operatorname{odd} \ell: \varphi(\ell) \mid n\} .
$$

Note that $S_{1} \subset S_{2} \subset S_{3}$ and $S_{3}$ depends only on $n$, not on $d$.

If $\ell$ is odd, $\ell \mid d$, and $\ell \notin S_{1}$, then $G_{\ell} / H^{+}$is non-trivial. Thus choosing a suitable $\psi$, we may arrange that the conductor of $\chi_{1}=\chi_{0} \psi$ is divisible by every prime dividing $d$ which is not in $S_{1}$.

For the odd primes $\ell$ which divide $d$ and do not divide the conductor of $\chi_{1}$ (a subset of $S_{1}$, thus also a subset of $S_{3}$ ), we must arrange that $\chi^{\prime}(\ell) \neq 1$ (where $\chi^{\prime}$ is the primitive character inducing $\chi)$.

Recall that $G_{\ell} / H^{+}$is cyclic. We now remark that if $C$ is a cyclic group and $a \in C$, and $z \in \mathbb{C}$, then the set of characters $\psi: C \rightarrow \mathbb{C}$ such that $\chi(a) \neq z$ has cardinality at least $|C|(1-1 /|\langle a\rangle|)$ (where $|\langle a\rangle|$ is the order of $a$ ). If we have several elements $a_{1}, \ldots, a_{n}$ and several values $z_{1}, \ldots, z_{n}$ to avoid, then the number of characters $\psi$ such that $\psi\left(a_{i}\right) \neq z_{i}$ is at least

$$
|C|\left(1-\frac{1}{\left|\left\langle a_{1}\right\rangle\right|}-\cdots-\frac{1}{\left|\left\langle a_{n}\right\rangle\right|}\right) .
$$

Thus we can find such a character provided that each $a_{i}$ has order $>n$. 
Now we use that $d$ is large to conclude that a large prime power $\ell^{e}$ divides $d$. (Note that $\ell$ might be 2 here.) Then $G_{\ell} / H^{+}$is a cyclic group in which the order of each prime in $S_{1}$ is large. (The primes in $S_{1}$ are also in $S_{3}$, so belong to a set fixed independently of $d$.) We want a character $\psi$ of $G_{\ell} / H^{+}$which satisfies $\psi(r) \neq \chi_{1}^{-1}(r)$ for all $r \in S_{1}$. We also want $\psi \chi_{1}$ to have non-trivial $\ell$ component which, phrased in the language above, means that we want $\psi(a) \neq 1$ for some fixed generator of $G_{\ell} / H^{+}$. Since the size of $S_{1}$ is fixed depending only on $n$, the discussion of the previous paragraph shows that these conditions can be met if $\ell^{e}$ is large enough.

Setting $\chi=\psi \chi_{1}$ with $\psi$ as in the previous paragraph yields a character $\chi$ such that $c_{\chi} \neq 0$, and this completes the proof.

Proof of Theorem 3.2. We retain the concepts and notation of the proof of Theorem 3.1. We also say that a subgroup of order 2 is "exceptional" if it does not contain -1 or $\frac{1}{2} d+1$.

Since $n=2$, the set $S_{3}=\{3\}$ and the set $S_{2}$ is either empty (if $3 \not d$ or $9 \mid d$ ) or $S_{2}=\{3\}$ (if 3 exactly divides $d$ ). If $S_{2}$ is empty and $H$ is an exceptional subgroup of order 2 , then the first part of the proof of Theorem 3.1 provides a primitive odd character trivial on $H$, and so $H$ is not balanced.

Suppose we are in the case where 3 exactly divides $d$. Following the first part of the proof of Theorem 3.1, we have a character $\chi_{1}$ of $G$ with conductor divisible by $d^{\prime}=d / 3$ which is odd, trivial on $H$, and, if $4 \mid d$, satisfies $\chi_{1}\left(\frac{1}{2} d+1\right)=-1$. If the conductor of $\chi_{1}$ is $d$ or if the primitive character $\chi^{\prime}$ inducing $\chi_{1}$ has $\chi^{\prime}(3) \neq 1$, then setting $\chi=\chi_{1}$ we have $c_{\chi} \neq 0$ and we see that $H$ is not balanced.

If not, we will modify $\chi_{1}$. Note that if $\ell=2$ and $16 \mid d$, or $\ell=5$ and $25 \mid d$, or $\ell$ is a prime $\geq 7$ and $\ell \mid d$, then the order of 3 in $G_{\ell} / H^{+}$is at least 3. Thus in these cases, there is a character $\psi$ of $G_{\ell} / H^{+}$so that the $\ell$ part of $\chi=\chi_{1} \psi$ is non-trivial and so that the primitive character $\chi^{\prime}$ inducing $\chi$ satisfies $\chi^{\prime}(3) \neq 1$. Then $c_{\chi} \neq 0$ and $H$ is not balanced.

This leaves a small number of values of $d$ to check for exceptional balanced subgroups of order 2. Namely, we just need to check divisors of $8 \cdot 3 \cdot 5=120$ which are divisible by 3 . A quick computation which we leave to the reader finishes the proof.

\section{Distribution OF NUMBers $d$ With $\langle p\rangle_{d}$ BALANCED}

Fix an integer $p$ with $|p|>1$. In our application to elliptic curves, $p$ is an odd prime number, but it seems interesting to state our results on balanced subgroups in a more general context. Let $\mathcal{B}_{p}$ denote the set of integers $d>2$ coprime to $p$ for which $\langle p\rangle_{d}$ is a balanced subgroup of $\mathbb{U}_{d}$. Further, let

$$
\begin{aligned}
& \mathcal{B}_{p, 0}=\left\{d>2:(d, p)=1,4 \mid d, \frac{1}{2} d+1 \in\langle p\rangle_{d}\right\}, \\
& \mathcal{B}_{p, *}=\mathcal{B}_{p} \backslash \mathcal{B}_{p, 0}, \\
& \mathcal{B}_{p, 1}=\left\{d>2:(d, p)=1,-1 \in\langle p\rangle_{d}\right\} .
\end{aligned}
$$

Note that if $p$ is even then $\mathcal{B}_{p, 0}$ is empty. For any set $\mathcal{A}$ of positive integers and $x$ a real number at least 1 , we let $\mathcal{A}(x)=|\mathcal{A} \cap[1, x]|$.

We state the principal results of this section, which show that when $p$ is odd, most members of $\mathcal{B}_{p}$ lie in $\mathcal{B}_{p, 0}$. 
Theorem 4.1. For each odd integer $p$ with $|p|>1$, there are positive numbers $b_{p}, b_{p}^{\prime}$ with

$$
b_{p} \frac{x}{\log \log x} \leq \mathcal{B}_{p, 0}(x) \leq b_{p}^{\prime} \frac{x}{\log \log x}
$$

for all sufficiently large numbers $x$ depending on the choice of $p$.

Theorem 4.2. For each integer $p$ with $|p|>1$, there is a number $\epsilon_{p}>0$ such that for all $x \geq 3$,

$$
\mathcal{B}_{p, *}(x)=O_{p}\left(\frac{x}{(\log x)^{\epsilon_{p}}}\right) .
$$

We remark that $\mathcal{B}_{p, 1}$ has been studied by Moree. In particular we have the following result.

Theorem 4.3 ([9, Thm. 5]). For each integer $p$ with $|p|>1$ there are positive numbers $c_{p}, \delta_{p}$ such that

$$
\mathcal{B}_{p, 1}(x) \sim c_{p} \frac{x}{(\log x)^{\delta_{p}}}, x \rightarrow \infty
$$

Note that for $p$ prime we have $\delta_{p}=\frac{2}{3}$. We believe that $\mathcal{B}_{p, 0}$ and $\mathcal{B}_{p, 1}$ comprise most of $\mathcal{B}_{p}$ and in fact we pose the following conjecture.

Conjecture 4.4. For each integer $p$ with $|p|>1$ we have

$$
\mathcal{B}_{p}(x)=\mathcal{B}_{p, 0}(x)+(1+o(1)) \mathcal{B}_{p, 1}(x), x \rightarrow \infty,
$$

that is, $\mathcal{B}_{p, *}(x) \sim \mathcal{B}_{p, 1}(x)$ as $x \rightarrow \infty$.

It is easy to see that $\mathcal{B}_{p, 1} \cap \mathcal{B}_{p, 0}$ has at most one element. Indeed, the cyclic group $\langle p\rangle_{d}$ has at most one element of order exactly 2, so if $d \in \mathcal{B}_{p, 1} \cap \mathcal{B}_{p, 0}$, then for some $f$ we have $p^{f} \equiv$ $-1 \equiv \frac{1}{2} d+1(\bmod d)$ and this can happen only when $d=4$. This at least shows that $\mathcal{B}_{p}(x) \geq$ $\mathcal{B}_{p, 0}(x)+(1+o(1)) \mathcal{B}_{p, 1}(x)$ as $x \rightarrow \infty$.

We now begin a discussion leading to the proofs of Theorems 4.1 and 4.2. The following useful result comes from [4, Theorem 2.2].

Proposition 4.5. There is an absolute positive constant c such that for all numbers $x \geq 3$ and any set $\mathcal{R}$ of primes in $[1, x]$, the number of integers in $[1, x]$ not divisible by any member of $\mathcal{R}$ is at most

$$
c x \prod_{r \in \mathcal{R}}\left(1-\frac{1}{r}\right) \leq c x \exp \left(-\sum_{r \in \mathcal{R}} \frac{1}{r}\right) .
$$

Note that the inequality in the display follows immediately from the inequality $1-\theta<\mathrm{e}^{-\theta}$ for every $\theta \in(0,1)$.

For a positive integer $m$ coprime to $p$, recall that $l_{p}(m)$ denotes the order of $\langle p\rangle_{m}$. If $r$ is a prime, we let $v_{r}(m)$ denote that integer $v$ with $r^{v} \mid m$ and $r^{v+1} \not \backslash m$.

We would like to give a criterion for membership in $\mathcal{B}_{p, 0}$, but before this, we establish an elementary lemma.

Lemma 4.6. Let $p$ be an odd integer with $|p|>1$ and let $k, i$ be positive integers. Then

$$
v_{2}\left(\frac{p^{2^{i} k}-1}{p^{2 k}-1}\right)=i-1
$$


Proof. The result is clear if $i=1$. If $i>1$, we see that

$$
\frac{p^{2^{i} k}-1}{p^{2 k}-1}=\left(p^{2 k}+1\right)\left(p^{4 k}+1\right) \ldots\left(p^{2^{i-1} k}+1\right),
$$

which is a product of $i-1$ factors that are each $2(\bmod 4)$.

The following result gives a criterion for membership in $\mathcal{B}_{p, 0}$.

Proposition 4.7. Let $p$ be odd with $|p|>1$ and let $m \geq 1$ be an odd integer coprime to $p$. If $l_{p}(m)$ is odd, then $2^{j} m \in \mathcal{B}_{p, 0}$ if and only if $j=1+v_{2}(p-1)$ or $j>v_{2}\left(p^{2}-1\right)$. If $l_{p}(m)$ is even, then $2^{j} m \in \mathcal{B}_{p, 0}$ if and only if $j>v_{2}\left(p^{l_{p}(m)}-1\right)$.

Proof. We first prove the "only if" part. Assume that $d=2^{j} m \in \mathcal{B}_{p, 0}$ and let $f$ be an integer with $p^{f} \equiv \frac{1}{2} d+1(\bmod d)$. Then $l_{p}(m) \mid f$ so that $j-1=v_{2}\left(p^{f}-1\right) \geq v_{2}\left(p^{l_{p}(m)}-1\right)$. This establishes the "only if" part if $l_{p}(m)$ is even, and it also shows that $j \geq 1+v_{2}(p-1)$ always, so in particular if $l_{p}(m)$ is odd. Suppose $l_{p}(m)$ is odd and $1+v_{2}(p-1)<j \leq v_{2}\left(p^{2}-1\right)$. Then $j-1>v_{2}(p-1)$, so that $l_{p}\left(2^{j-1} m\right)$ is even. Using $l_{p}(m)$ odd, this implies that $2 l_{p}(m) \mid f$, so that $2^{j}\left|\left(p^{2}-1\right)\right|\left(p^{f}-1\right)$, contradicting $p^{f} \equiv \frac{1}{2} d+1(\bmod d)$.

Towards showing the "if" part, let $v=v_{2}\left(p^{l_{p}(m)}-1\right)$. We have $p^{l_{p}(m)}-1 \equiv 2^{v} m\left(\bmod 2^{v+1} m\right)$, so that $2^{v+1} m \in \mathcal{B}_{p, 0}$. If $j>v+1$ and $l_{p}(m)$ is even, then with $f=2^{j-v-1} l_{p}(m)$, Lemma 4.6 implies that $p^{f}-1 \equiv 2^{j-1} m\left(\bmod 2^{j} m\right)$, so that $2^{j} m \in \mathcal{B}_{p, 0}$. If $l_{p}(m)$ is odd, then $v=v_{2}(p-1)$, so that $2^{v+1} \in \mathcal{B}_{p, 0}$. Finally assume that $j>v_{2}\left(p^{2}-1\right)$ and $l_{p}(m)$ is odd. Then Lemma4.6implies that $p^{2^{j-v_{2}\left(p^{2}-1\right)} l_{p}(m)}-1 \equiv 2^{j-1} m\left(\bmod 2^{j} m\right)$, so that $2^{j} m \in \mathcal{B}_{p, 0}$. This concludes the proof.

Proof of Theorem 4.1. For $m \geq 1$ coprime to $2 p$, let

$$
f_{p}(m):=v_{2}\left(p^{l_{p}(m)}-1\right), \quad f_{p}^{\prime}(m):=\max \left\{f_{p}(m), v_{2}\left(p^{2}-1\right)\right\} .
$$

Proposition 4.7 implies that if $2^{j} m \in \mathcal{B}_{p, 0}$ with $m$ odd, then $j>f_{p}(m)$. Further, if $(m, 2 p)=1$ then $2^{j} m \in \mathcal{B}_{p, 0}$ for all $j>f_{p}^{\prime}(m)$.

Using this last property, we have $\mathcal{B}_{p, 0}(x)$ at least as big as the number of choices for $m$ coprime to $2 p$ with $1<m \leq x / 2^{f_{p}^{\prime}(m)+1}$. Thus, the lower bound in the theorem will follow if we show that there are at least $b_{p} x / \log \log x$ integers $m$ coprime to $2 p$ with $m \leq x / 2^{f_{p}^{\prime}(m)+1}$.

Let $\lambda(m)$ denote Carmichael's function at $m$, which is the order of the largest cyclic subgroup of $\mathbb{U}_{m}$. Then $l_{p}(m) \mid \lambda(m)$. Also, for $m>2, \lambda(m)$ is even, so that

$$
f_{p}^{\prime}(m) \leq g_{p}(m):=v_{2}\left(p^{\lambda(m)}-1\right) .
$$

Thus, the lower bound in the theorem will follow if we show that there are at least $b_{p} x / \log \log x$ integers $m$ coprime to $2 p$ with $m \leq x / 2^{g_{p}(m)+1}$. Using Lemma 4.6, we have $g_{p}(m)+1=$ $v_{2}(\lambda(m))+v_{2}\left(p^{2}-1\right)$. Further, it is easy to see that $2^{v_{2}\left(p^{2}-1\right)} \leq 2(|p|+1)$, with equality when $|p|+1$ is a power of 2 .

It follows from [10, Section 2, Remark 1] that uniformly for all $x \geq 3$ and all positive integers $n$,

$$
\sum_{\substack{r \leq x \\ r \text { prime } \\ n \mid r-1}} \frac{1}{r}=\frac{\log \log x}{\varphi(n)}+O\left(\frac{\log (2 n)}{\varphi(n)}\right) .
$$


We apply this with $n=2^{g_{0}+1}$, where $g_{0}$ is the first integer with $2^{g_{0}} \geq 4 \log \log x$. Thus, if $\mathcal{R}$ is the set of primes $r \leq x$ with $v_{2}(r-1)>g_{0}$, we have for $x$ sufficiently large,

$$
\sum_{r \in \mathcal{R}} \frac{1}{r}<\frac{1}{3}
$$

Let $z=x /(25|p| \log \log x)$. In $[1, z]$ there are $(\varphi(|p|) /(2|p|)) z+O_{p}(1)$ integers coprime to $2 p$. And for a given value of $r \in \mathcal{R}$ there are at most $(\varphi(|p|) /(2|p|)) z / r+O_{p}(1)$ numbers in $[1, z]$ coprime to $2 p$ and divisible by $r$. It follows that for $x$ sufficiently large depending on the choice of $p$, there are at least

$$
\frac{\varphi(|p|)}{2|p|} z-\frac{\varphi(|p|)}{2|p|} z \sum_{r \in \mathcal{R}} \frac{1}{r}+O_{p}\left(\sum_{r \in \mathcal{R}} 1\right)>\frac{\varphi(|p|)}{4|p|} z
$$

integers $m \leq z$ coprime to $2 p$ and not divisible by any prime $r \in \mathcal{R}$. (We used that $|\mathcal{R}|=$ $O(x / \log x)$ to estimate the $O$-term above.)

It remains to note that if $m \leq z, m$ is coprime to $2 p$, and $m$ is not divisible by any prime in $\mathcal{R}$, then $v_{2}(\lambda(m)) \leq g_{0}$, so that

$$
2^{g_{p}(m)+1} \leq 2^{v_{2}(\lambda(m))+v_{2}\left(p^{2}-1\right)} \leq 2^{g_{0}} 2^{v_{2}\left(p^{2}-1\right)} \leq 2^{g_{0}} \cdot 2(|p|+1) \leq 2^{g_{0}} \cdot 3|p|<25|p| \log \log x .
$$

Thus, $2^{g_{p}(m)+1} m \in \mathcal{B}_{p, 0}$ and $2^{g_{p}(m)+1} m \leq x$, so that

$$
\mathcal{B}_{p, 0}(x) \geq \frac{\varphi(|p|)}{100 p^{2}} \frac{x}{\log \log x}
$$

for $x$ sufficiently large depending on the choice of $p$. This completes our proof of the lower bound.

For the upper bound, it suffices to show that

$$
N(x):=\mathcal{B}_{p, 0}(x)-\mathcal{B}_{p, 0}(x / 2)=O_{p}\left(\frac{x}{\log \log x}\right) .
$$

(With this assumption, no two numbers $d$ counted can have the same odd part.) We shall assume that $p$ is not a square, the case when $p=p_{0}^{2^{j}}$ for some integer $p_{0}$ and $j \geq 1$ being only slightly more complicated. From Proposition 4.7, $N(x)$ is at most the number of odd numbers $m$ coprime to $p$ with $m \leq x / 2^{f_{p}(m)+1}$. Let $N_{k}(x)$ be the number of odd numbers $m \leq x / 2^{k+1}$ with $m$ coprime to $p$ and $f_{p}(m)=k$. Then

$$
N(x)=\sum_{k} N_{k}(x)=\sum_{2^{k} \leq \log \log x} N_{k}(x)+O\left(\frac{x}{\log \log x}\right) .
$$

We now concentrate our attention on $N_{k}(x)$ with $2^{k} \leq \log \log x$. If $f_{p}(m)=k$, then $m$ is not divisible by any prime $r$ with $(p / r)=-1$ and $2^{k+1} \mid r-1$. Then, using (4.8) and quadratic reciprocity,

$$
\sum_{\substack{r \leq x \\(p / r)=-1 \\ 2^{k+1} \mid r-1 \\ r \text { prime }}} \frac{1}{r}=\frac{\log \log x}{2^{k+1}}+O_{p}\left(\frac{k}{2^{k}}\right) .
$$


By Propostion 4.5, the number of integers $m \leq x / 2^{k+1}$ not divisible by any such prime $r$ is at most

$$
O\left(\frac{x}{2^{k+1}} \exp \left(-\sum_{r} \frac{1}{r}\right)\right)=O_{p}\left(\frac{x}{2^{k+1}} \exp \left(-\frac{\log \log x}{2^{k+1}}\right)\right) .
$$

Summing this expression for $2^{k} \leq \log \log x$ gives $O_{p}(x / \log \log x)$, which completes the proof of Theorem 4.1.

Remark 4.9. One might wonder if there is a positive constant $\beta_{p}$ such that if $p$ is odd with $|p|>1$, then $\mathcal{B}_{p, 0}(x) \sim \beta_{p} x / \log \log x$ as $x \rightarrow \infty$. Here we sketch an argument that no such $\beta_{p}$ exists; that is,

$$
0<\liminf _{x \rightarrow \infty} \frac{\mathcal{B}_{p, 0}(x)}{x / \log \log x}<\limsup _{x \rightarrow \infty} \frac{\mathcal{B}_{p, 0}(x)}{x / \log \log x}<\infty .
$$

First note that but for $O_{p}\left(x /(\log x)^{1 / 2}\right)$ values of $d \leq x$ there is a prime $r \mid d$ with $(p / r)=-1$. (We are assuming here that $p$ is not a square.) For such values of $d=2^{j} m$, with $m$ odd, we have $2 \mid l_{p}(m)$, so that in the notation above we have $f_{p}(m)=f_{p}^{\prime}(m) \geq 3$. Thus it suffices to count numbers $2^{j} m \leq x$ with $m$ odd and $j>f_{p}(m) \geq 3$. Note that

$$
f_{p}(m)=v_{2}\left(l_{p}(m)\right)+v_{2}\left(p^{2}-1\right)-1=v_{2}\left(l_{p}(m)\right)+h_{p}-1,
$$

say. Further,

$$
v_{2}\left(l_{p}(m)\right)=\max _{r \mid m} v_{2}\left(l_{p}(r)\right)
$$

where $r$ runs over the prime divisors of $m$. We have

$$
\begin{aligned}
\left\{r \text { prime }: v_{2}\left(l_{p}(r)\right)=k\right\} & = \\
\bigcup_{i \geq 0}\left\{r \text { prime }: v_{2}(r-1)\right. & \left.=k+i, p \text { is a } 2^{i} \text { power }(\bmod r) \text { and not a } 2^{i+1} \text { power }(\bmod r)\right\} .
\end{aligned}
$$

For $k>(\log \log \log x)^{2}$, the density of primes $r \equiv 1\left(\bmod 2^{k}\right)$ is so small that we may assume that no $d$ is divisible by such a prime $r$. For $k$ below this bound, the density of primes $r$ with $v_{2}\left(l_{p}(r)\right)=k$ is $1 /\left(3 \cdot 2^{k-1}\right)$. Thus, there is a positive constant $c_{k, p}$ with $c_{k, p} \rightarrow 1$ as $k \rightarrow \infty$ such that the density of integers $m$ coprime to $2 p$ and with $f_{p}(m)<k+h_{p}$ is asymptotically equal to

$$
c_{p}(\varphi(2|p|) /(2|p|)) \exp \left(-(\log \log x) /\left(3 \cdot 2^{k}\right)\right),
$$

as $x \rightarrow \infty$. Thus, the number of $m \leq x / 2^{k+h_{p}}$ coprime to $2 p$ and with $f_{p}(m)=k+h_{p}-1$ is asymptotically equal to

$$
c_{k, p} \frac{\varphi(2|p|)}{2|p|} \frac{x}{2^{k+h_{p}}} \frac{\log \log x}{3 \cdot 2^{k}} \exp \left(-\frac{\log \log x}{3 \cdot 2^{k}}\right)
$$

as $x \rightarrow \infty$. This expression then needs to be summed over $k$. For $k$ small, the count is negligible because of the exp factor. For $k$ larger, we can assume that the coefficients $c_{k, p}$ are all 1 , and then the sum takes on the form

$$
\frac{\varphi(2|p|)}{2|p| 2^{h_{p}}} \frac{x}{\log \log x} \sum_{k} \frac{(\log \log x)^{2}}{3 \cdot 2^{2 k}} \exp \left(-\frac{\log \log x}{3 \cdot 2^{k}}\right) .
$$

Letting this sum on $k$ be denoted $g(x)$, it remains to note that $g(x)$ is bounded away from both 0 and $\infty$ yet does not tend to a limit, cf. [5, Theorem 3.25]. 
To prove Theorem 4.2, we first establish the following result.

Proposition 4.10. Let $p$ be an integer with $|p|>1$. Let $d$ be a positive integer coprime to $p$ such that $d$ is divisible by odd primes $s, t$ with

$$
l_{p}(s) \equiv 2(\bmod 4), \quad l_{p}(t) \equiv 1(\bmod 2), \quad\langle p,-1\rangle_{s} \neq \mathbb{U}_{s}, \quad\langle p,-1\rangle_{t} \neq \mathbb{U}_{t} .
$$

Assume that $4 \mid l_{p}(d)$. Then either $4 \mid d$ and $\frac{1}{2} d+1 \in\langle p\rangle_{d}$ or $\langle p\rangle_{d}$ is not balanced.

Proof. Let $k=l_{p}(d)$. First assume that $4 \mid d$ and $\frac{1}{2} d+1 \notin\langle p\rangle_{d}$. Let $2^{\kappa}$ be the largest power of 2 in $k$. Write $d=2^{j} m$ where $m$ is odd, let $2^{\kappa_{1}}$ be the power of 2 in $l_{p}(m)$, and let $2^{\kappa_{2}}=l_{p}\left(2^{j}\right)$. Then $\kappa=\max \left\{\kappa_{1}, \kappa_{2}\right\}$. Suppose that $\kappa_{2}>\kappa_{1}$. We have $p^{k / 2} \equiv 1(\bmod m)$ and $p^{k / 2} \not \equiv 1\left(\bmod 2^{j}\right)$. Since $4 \mid k$, we have $p^{k / 2}+1 \equiv 2(\bmod 4)$, and since $p^{k}-1=\left(p^{k / 2}-1\right)\left(p^{k / 2}+1\right)$, we have $p^{k / 2} \equiv 1\left(\bmod 2^{e-1}\right)$. Thus, $p^{k / 2} \equiv \frac{1}{2} d+1(\bmod d)$, contrary to our assumption. Hence, we may assume that $\kappa=\kappa_{1} \geq \kappa_{2}$. Note that this inequality holds too in the case that $4 \backslash \chi d$, since then, $\kappa_{2}=0$.

We categorize the odd prime powers $r^{a}$ coprime to $p$ as follows.

- Type 1: $\langle p,-1\rangle_{r^{a}}=\mathbb{U}_{r^{a}}$.

- Type 2: $\langle p,-1\rangle_{r^{a}} \neq \mathbb{U}_{r^{a}}$.

- Type 3: It is Type 2 and also $l_{p}\left(r^{a}\right) \equiv 2(\bmod 4)$.

- Type 4: It is Type 2 and also $l_{p}\left(r^{a}\right)$ is odd.

By assumption $d$ has at least one Type 3 prime power component and at least one Type 4 prime power component. We will show that $\langle p\rangle_{d}$ is not balanced in $\mathbb{U}_{d}$. By Proposition 2.4, it is sufficient to exhibit an odd character $\chi(\bmod d)$ that is trivial at $p$ with conductor $d^{\prime}$ divisible by the same odd primes as are in $d$, and with either $d \equiv 2(\bmod 4)$ or $d / d^{\prime}$ odd.

Let $r_{1}^{a_{1}} \| d$ where the power of 2 in $l_{p}\left(r_{1}^{a_{1}}\right)$ is $2^{\kappa_{1}}$. (Note that $r_{1}^{a_{1}}$ cannot be Type 3 nor Type 4, since we have $\kappa_{1}=\kappa \geq 2$, so that $4 \mid l_{p}\left(r_{1}^{a_{1}}\right)$.) Consider the Type 1 prime powers in $d$, other than possibly $r_{1}^{a_{1}}$ in case it is of Type 1 . For each we take the quadratic character and we multiply these together to get a character $\chi_{1}$ whose conductor contains all of the primes involved in Type 1 prime powers, except possibly $r_{1}$.

If $j \leq 1$, we let $\psi_{2^{j}}$ be the principal character $\bmod 2^{j}$. If $j \geq 2$, let $\psi_{2^{j}}$ be a primitive character $\bmod 2^{j}$ with $\psi_{2^{j}}(p)=\zeta$, a primitive $2^{\kappa_{2}}$-th root of unity. Let $\chi_{2}=\chi_{1} \psi_{2^{j}}$.

We choose a character $\psi_{r_{1}^{a_{1}}} \bmod r_{1}^{a_{1}}$ with $\psi_{r_{1}^{a_{1}}}(p)=\chi_{2}(p)^{-1}$ if $\chi_{2}(p) \neq 1$, and otherwise we choose it so that $\psi_{r_{1}}^{a_{1}}(p)=-1$. Thus, this character is non-principal. Let $\chi_{3}=\psi_{r_{1}^{a_{1}}} \chi_{2}$. We now have $\chi_{3}(p)= \pm 1$.

If $\chi_{3}(p)=-1$ we use a Type 3 prime power $r_{3}^{a_{3}} \| d$ and choose a character $\psi_{r_{3}^{a_{3}}}\left(\bmod r_{3}^{a_{3}}\right)$ with $\psi_{r_{3} a_{3}}(p)=-1$. Let $\chi_{4}=\chi_{3} \psi_{r_{3} a_{3}}$. If $\chi_{3}(p)=1$, we let $\chi_{4}=\chi_{3}$. We now have $\chi_{4}(p)=1$.

If $\chi_{4}(-1)=1$, we use a Type 4 prime power $r_{4}^{a_{4}} \| d$ and choose a character $\psi_{r_{4}}^{a_{4}}\left(\bmod r_{4}^{a_{4}}\right)$ with $\psi_{r_{4} a_{4}}(p)=1$ and $\psi_{r_{4} a_{4}}(-1)=-1$. Let $\chi_{5}=\chi_{4} \psi_{r^{a}}$. If $\chi_{4}(-1)=-1$, we let $\chi_{5}=\chi_{4}$.

All remaining prime powers $r^{a}$ in $d$ are of Type 2. For these we take non-principal characters that are trivial on $\langle p,-1\rangle_{r^{a}}$, and multiply them in to $\chi_{5}$ to form $\chi_{6}$. This is the character we are looking for, and so $\langle p\rangle_{d}$ is not balanced. This completes our proof.

Proof of Theorem 4.2. In the proof we shall assume that $p$ is neither a square nor twice a square, showing in these cases that we may take $\epsilon_{p}=1 / 16$. The remaining cases are done with small adjustments to the basic argument, but may require a smaller value for $\epsilon_{p}$. 
Let $d \leq x$ be coprime to $p$. The set of primes $r \nmid p$ with $r \equiv 1(\bmod 4)$ and for which $p$ is a quadratic nonresidue has density $1 / 4$, and in fact, the sum of reciprocals of such primes $r \leq x$ is $\frac{1}{4} \log \log x+O_{p}(1)$. (This follows from either (4.8) and quadratic reciprocity or from the Chebotarev density theorem.) Thus by Proposition 4.5, the number of integers $d \leq x$ not divisible by any of these primes $r$ is $O_{p}\left(x /(\log x)^{1 / 4}\right)$. Thus, we may assume that $d$ is divisible by such a prime $r$ and so that $4 \mid l_{p}(d)$.

Note that if $r \equiv 5(\bmod 8)$ and that $p$ is a quadratic residue modulo $r$, but not a fourth power, then any $r^{a}$ is of Type 3 . The density of these primes $r$ is $1 / 16$, by the Chebotarev theorem, in fact, the sum of reciprocals of such primes $r \leq x$ is $\frac{1}{16} \log \log x+O_{p}(1)$. So the number of values of $d \in[3, x]$ not divisible by at least one of them is $O_{p}\left(x /(\log x)^{1 / 16}\right)$, using Proposition 4.5. Also note that if $r \equiv 5(\bmod 8)$ and $p$ is a nonzero fourth power modulo $r$, then any $r^{a}$ is Type 4 . The density of these primes $r$ is also $1 / 16$, and again the number of $d \in[3, x]$ not divisible by at least one of them is $O_{p}\left(x /(\log x)^{1 / 16}\right)$.

Thus, the number of values of $d \leq x$ coprime to $p$ and not satisfying the hypotheses of Proposition 4.10 is $O\left(x /(\log x)^{1 / 16}\right)$. This completes the proof of Theorem 4.2.

\section{THE AVERAGE AND NORMAL ORDER OF THE RANK}

In this section we consider the average and normal order of the rank of the curve $E_{d}$ given in Theorem 1.1 as $d$ varies.

It is clear from Theorem 1.1 that for $q$ odd,

$$
\operatorname{Rank} E_{d}\left(\mathbb{F}_{q}(u)\right) \leq \begin{cases}d-2 & \text { if } d \text { is even } \\ d-1 & \text { if } d \text { is odd }\end{cases}
$$

with equality when $d \in \mathcal{B}_{p, 1}$ and $q \equiv 1(\bmod d)$.

For all $q$ and $d>1$, it is known [1, Prop. 6.9] that

$$
\operatorname{Rank} E_{d}\left(\mathbb{F}_{q}(u)\right) \leq \frac{d}{2 \log _{q} d}+O\left(\frac{d}{\left(\log _{q} d\right)^{2}}\right) .
$$

(Here $\log _{q} d$ is the $\log$ arithm of $d$ base $q$, i.e., $\log d / \log q$.) We do not include the details here, but this bound can be proved directly for the the curves in Theorem 1.1 using that theorem. In addition, for $q$ odd, considering values of $d$ of the form $q^{f}+1$ for some positive integer $f$, and using Theorem 1.1, we see that the main term in this inequality is sharp for this family of curves.

We show below that although the average rank of $E_{d}\left(\mathbb{F}_{q}(u)\right)$ is large-its average for $d$ up to $x$ is at least $x^{1 / 2}$-for "most" values of $d$ the rank is much smaller.

Theorem 5.1. There is an absolute constant $\alpha>\frac{1}{2}$ with the following property. For each odd prime $p$ and finite field $\mathbb{F}_{q}$ of characteristic $p$, with $\mathbb{F}_{q}(u)$ and $E_{d}$ as in Theorem 1.1 we have

$$
x^{\alpha} \leq \frac{1}{x} \sum_{d \leq x} \operatorname{Rank} E_{d}\left(\mathbb{F}_{q}(u)\right) \leq x^{1-\log \log \log x /(2 \log \log x)}
$$

for all sufficiently large $x$ depending on the choice of $p$.

Proof. This result follows almost immediately from [11, Theorem 1]. A result is proved there for the average value of the rank of curves in a different family also parametrized by a positive integer 
$d$. Using the notation from the present paper, if $d \in \mathcal{B}_{p, 1}$ the rank of the curve considered in [11] is within 4 of

$$
\sum_{\substack{e \mid d \\ e>2}} \frac{\varphi(e)}{l_{q}(e)}
$$

We have $d \in \mathcal{B}_{p, 1}$ implies that $e \in \mathcal{B}_{p, 1}$ for all $e \mid d$ with $e>2$. By Theorem 1.1, formula (5.2) is exactly the rank of $E_{d}\left(\mathbb{F}_{q}(u)\right)$ for $d \in \mathcal{B}_{p, 1}$. Since the proof of the lower bound $x^{\alpha}$ in [11] uses only values of $d \in \mathcal{B}_{p, 1}$, we have the lower bound $x^{\alpha}$ in the present theorem.

Since the rank of $E_{d}\left(\mathbb{F}_{q}(u)\right)$ is bounded above by the formula (5.2) whether or not $d$ is in $\mathcal{B}_{p, 1}$, and in fact whether or not $\langle p\rangle_{d}$ is balanced, the argument given in [11] for the upper bound gives our upper bound here.

Theorem 5.3. For each odd prime $p$ and finite field $\mathbb{F}_{q}$ of characteristic $p$, with $\mathbb{F}_{q}(u)$ and $E_{d}$ as in Theorem 1.1 we have but for $o(x / \log \log x)$ values of $d \leq x$ with $d \in \mathcal{B}_{p}$ that

$$
\operatorname{Rank} E_{d}\left(\mathbb{F}_{q}(u)\right) \geq(\log d)^{(1+o(1)) \log \log \log d}
$$

as $x \rightarrow \infty$. Further, assuming the GRH, we have but for $o(x / \log \log x)$ values of $d \leq x$ with $d \in \mathcal{B}_{p}$ that

$$
\operatorname{Rank} E_{d}\left(\mathbb{F}_{q}(u)\right) \leq(\log d)^{(1+o(1)) \log \log \log d}
$$

as $x \rightarrow \infty$. Assuming the GRH, this upper bound holds but for $o(x)$ values of $d \leq x$ coprime to $p$ as $x \rightarrow \infty$, regardless of whether $d \in \mathcal{B}_{p}$.

Proof. For $d \in \mathcal{B}_{p}$, Theorem 1.1 implies that the rank of $E_{d}\left(\mathbb{F}_{q}(u)\right)$ is at least $\varphi(d) / l_{q}(d) \geq$ $\varphi(d) / \lambda(d)$, where $\lambda$ was defined in the previous section as the order of the largest cyclic subgroup of $\mathbb{U}_{d}$. It is shown in the proof of Theorem 2 in [3] that on a set of asymptotic density 1 , we have $\varphi(d) / \lambda(d)=(\log d)^{(1+o(1)) \log \log \log d}$. We would like to show this holds for almost all $d \in \mathcal{B}_{p}$. Note that we have $\varphi(m) / \lambda(m)=(\log m)^{(1+o(1)) \log \log \log m}$ for almost all odd numbers $m$. We have for all odd $m$ and every integer $j \geq 0$ that

$$
\frac{\varphi(m)}{\lambda(m)} \leq \frac{\varphi\left(2^{j} m\right)}{\lambda\left(2^{j} m\right)} \leq 2^{j} \frac{\varphi(m)}{\lambda(m)}
$$

Thus, for almost all odd numbers $m$ we have for all nonnegative integers $j$ with $2^{j} \leq \log m$ that $\left.\varphi\left(2^{j} m\right) / \lambda\left(2^{j} m\right)\right)=\left(\log \left(2^{j} m\right)\right)^{(1+o(1)) \log \log \log \left(2^{j} m\right)}$. Further, it follows from (4.8) that but for a set of odd numbers $m$ of asymptotic density 0 , we have $v_{2}(\lambda(m)) \leq 2 \log \log \log m$. It thus follows from Proposition 4.7 that for almost all odd numbers $m$ there is some nonnegative $j$ with $2^{j} m \in \mathcal{B}_{p}$ and $2^{j} \leq \log m$. By Theorems 4.1, 4.2 almost all members of $\mathcal{B}_{p}$ are of this form, and so we have the lower bound in the theorem.

For the upper bound we use an argument in [6]. There, Corollary 2 and the following remark imply that under the assumption of the GRH, for almost all numbers $d$ coprime to $p$ we have $\varphi(d) / l_{q}(d)=(\log d)^{(1+o(1)) \log \log \log d}$. We use that $\varphi(e) / l_{q}(e) \mid \varphi(d) / l_{q}(d)$ for $e \mid d$ and from the normal order of the number-of-divisors function $\tau(d)$, that most numbers $d$ have $\tau(d) \leq \log d$. It thus follows from Theorem 1.1 and the GRH that for almost all numbers $d$ coprime to $p$ that

$$
\operatorname{Rank} E_{d}\left(\mathbb{F}_{q}(u)\right) \leq \tau(d) \frac{\varphi(d)}{l_{q}(d)} \leq(\log d) \frac{\varphi(d)}{l_{q}(d)}=(\log d)^{(1+o(1)) \log \log \log d}
$$


We would like to show as well that this inequality continues to hold for almost all $d$ that are in $\mathcal{B}_{p}$. As above, the GRH implies that for almost all odd numbers $m$ coprime to $p$, we have $\varphi(m) / l_{q}(m)=(\log m)^{(1+o(1)) \log \log \log m}$. Since (5.4) continues to hold with $l_{q}$ in place of $\lambda$, it follows that for almost all odd $m$ and for all $j$ with $1 \leq 2^{j} \leq \log m$, that $\varphi\left(2^{j} m\right) / l_{q}\left(2^{j} m\right)=$ $\left(\log \left(2^{j} m\right)\right)^{(1+o(1)) \log \log \log \left(2^{j} m\right)}$. Again using the normal order of the number-of-divisors function $\tau$, we have that for almost all odd $m$ and all $j$ with $1 \leq 2^{j} \leq \log m$ that $\tau\left(2^{j} m\right) \leq \log m$. Further, as we noted above, from Theorems 4.1, 4.2, it follows that almost all members $d$ of $\mathcal{B}_{p}$ are of the form $2^{j} m$ with $m$ odd and $2^{j} \leq \log m$. The rank formula in Theorem 1.1 implies that the rank of $E_{d}\left(\mathbb{F}_{q}(u)\right)$ is bounded above by $\tau(d) \varphi(d) / l_{q}(d)$. Thus, for almost all $d \in \mathcal{B}_{p}$ we have the rank at most $(\log d)^{(1+o(1)) \log \log \log d}$. This completes the proof.

\section{REFERENCES}

[1] A. Brumer, The average rank of elliptic curves. I, Invent. Math. 109 (1992), 445-472.

[2] D. Ulmer et al., Explicit points on the Legendre curve III, In preparation.

[3] P. Erdős, C. Pomerance, and E. Schmutz, Carmichael's lambda function, Acta Arith. 58 (1991), 363-385.

[4] H. Halberstam and H.-E. Richert, Sieve methods, Academic Press, London, 1974.

[5] S. Li, On Artin's conjecture for composite moduli, Ph.D. thesis, U. Georgia, 1998.

[6] S. Li and C. Pomerance, On generalizing Artin's conjecture on primitive roots to composite moduli, J. Reine Angew. Math. 556 (2003), 205-224.

[7] D. Marcus, Number fields, Springer, New York, 1977.

[8] H. L. Montgomery and R. C. Vaughan, Multiplicative number theory I. Classical theory, Cambridge U. Press, Cambridge, 2007.

[9] P. Moree, On the divisors of $a^{k}+b^{k}$, Acta Arith. 80 (1997), 197-212.

[10] C. Pomerance, On the distribution of amicable numbers, J. Reine Angew. Math. 293/294 (1977), 217-222.

[11] C. Pomerance and I. E. Shparlinski, Rank statistics for a family of elliptic curves over a function field, Pure Appl. Math. Q., 6 (2010), 21-40.

[12] D. Ulmer, Explicit points on the Legendre curve, Preprint (2009).

Department of Mathematics, Dartmouth College, Hanover, NH 03755

E-mail address: carl.pomerance@dartmouth.edu

School of Mathematics, Georgia Institute of Technology, Atlanta, GA 30332

E-mail address: douglas.ulmer@math.gatech.edu 